\title{
The strength of the projective Martin conjecture
}

\author{
by \\ C. T. Chong (Singapore), Wei Wang (Guangzhou) and \\ Liang Yu (Nanjing)
}

\begin{abstract}
We show that Martin's conjecture on $\Pi_{1}^{1}$ functions uniformly $\leq_{T}$-order preserving on a cone implies $\Pi_{1}^{1}$ Turing Determinacy over ZF + DC. In addition, it is also proved that for $n \geq 0$, this conjecture for uniformly degree invariant $\Pi_{2 n+1}^{1}$ functions is equivalent over ZFC to $\boldsymbol{\Sigma}_{2 n+2}^{1}$-Axiom of Determinacy. As a corollary, the consistency of the conjecture for uniformly degree invariant $\Pi_{1}^{1}$ functions implies the consistency of the existence of a Woodin cardinal.
\end{abstract}

1. Introduction. A cone $C$ of reals with base $z$ is a set of the form $\left\{x \mid x \geq_{T} z\right\}$ where $\leq_{T}$ denotes Turing reducibility. A function $F: 2^{\omega} \rightarrow 2^{\omega}$ is degree invariant on $C$ if any two reals $x, y \geq_{T} z$ of the same Turing degree satisfy $F(x) \equiv_{T} F(y)$. The degree invariance is uniform on $C$ if there is a function $t$ such that if $x, y \geq_{T} z$, then $\Phi_{i}^{x}=y$ and $\Phi_{j}^{y}=x$ implies $\Phi_{m}^{F(x)}=F(y)$ and $\Phi_{n}^{F(y)}=F(x)$, where $t(i, j)=(m, n)$. The function $F$ is increasing on $C$ if $F(x) \geq_{T} x$ for all $x \geq z$, and order preserving on $C$ if $z \leq_{T} x \leq_{T} y$ implies $F(z) \leq_{T} F(x) \leq_{T} F(y)$. If this order preservation is witnessed by a function $t: \omega \rightarrow \omega$, i.e., $\Phi_{e}^{x}=y \geq_{T} z$ implies $\Phi_{t(e)}^{F(x)}=$ $F(y)$, then it is uniform (note that a uniformly order preserving function is necessarily uniformly degree invariant). Finally, given functions $F$ and $G$ degree invariant on a cone, write $F \geq_{M} G$ if $F(x) \geq_{\mathrm{T}} G(x)$ on a cone. Donald A. Martin conjectured that, under the assumption of ZF set theory plus the Axiom of Determinacy (AD) and Dependent Choice (DC):

(1) Every degree invariant function that is not increasing on a cone is a constant on a cone.

(2) $\leq_{M}$ prewellorders degree invariant functions which are increasing on a cone. Furthermore, if the $\leq_{M}$-rank of $F$ is $\alpha$, then $F^{\prime}$ has $\leq_{M}$-rank $\alpha+1$, where $F^{\prime}(x)=(F(x))^{\prime}$, the Turing jump of $F(x)$.

2010 Mathematics Subject Classification: 03D28, 03E35, 28A20.

Key words and phrases: Martin's conjecture, axiom of determinacy, Turing cone. 
Slaman and Steel [7] proved (1) for functions which are uniformly degree invariant on a cone and (2) for Borel functions which are increasing and order preserving. In [8] Steel showed (2) for uniformly degree invariant functions and conjectured that every function degree invariant on a cone is uniformly degree invariant on a cone.

While Martin [4] has shown that Borel determinacy is a theorem of $\mathrm{ZF}+\mathrm{DC}$ (hence conjectures (1) and (2) hold for $\boldsymbol{\Delta}_{1}^{1}$ functions that are uniformly degree invariant), it is known that $\mathrm{AD}$ in the analytical hierarchy beyond $\boldsymbol{\Delta}_{1}^{1}$ is a large cardinal axiom. An analysis of the proof in [8] shows that conjecture (2) for uniformly degree invariant $\boldsymbol{\Pi}_{2 n+1}^{1}$ functions follows from $\boldsymbol{\Delta}_{2 n+2}^{1}$ Determinacy. Thus a natural question for Martin's Conjectures (1) and (2) is their set-theoretic strength for uniformly degree invariant functions beyond $\boldsymbol{\Delta}_{1}^{1}$ in the analytical hierarchy. There is also a related question concerning the more restrictive uniformly order preserving functions, i.e. while (2) holds for such functions under AD according to Steel [8], the set-theoretic strength of (2) for these functions has not been considered.

A set of reals is degree invariant if it is closed under Turing equivalence. Martin [3] showed that under AD, every degree invariant set of reals either contains or is disjoint from a cone. By $\boldsymbol{\Pi}_{2 n+1^{-}}^{1}$ Turing Determinacy $\left(\boldsymbol{\Pi}_{2 n+1^{-}}^{1}\right.$ TD) we mean the assertion that every $\boldsymbol{\Pi}_{2 n+1}^{1}$ set of reals that is degree invariant either contains or is disjoint from a cone. We show in this paper that Conjecture (2) for uniformly order preserving $\Pi_{1}^{1}$ functions implies the existence of $0^{\#}$. Relativizing the argument to arbitrary reals $x$ leads to the conclusion that $x^{\#}$ exists for every $x$, so that by Harrington [1] we have the following theorem on the strength of Conjecture (2) for uniformly order preserving $\boldsymbol{\Pi}_{1}^{1}$ functions.

Main Theorem 1. If Conjecture (2) holds for uniformly order preserving $\boldsymbol{\Pi}_{1}^{1}$ functions then $\boldsymbol{\Pi}_{1}^{1}-T D$ is true.

We also show that in general, for $n \geq 0$, Conjecture (2) for uniformly degree invariant $\boldsymbol{\Pi}_{2 n+1}^{1}$ functions implies $\boldsymbol{\Sigma}_{2 n+2}^{1}$-TD, assuming $\boldsymbol{\Pi}_{2 n+1}^{1}$-uniformization when $n \geq 1$. In fact, by this, Steel [8] and an unpublished work of W. H. Woodin, we have the strength of Conjecture (2) for uniformly degree invariant $\Pi_{2 n+1}^{1}$ functions measured by $\boldsymbol{\Sigma}_{2 n+2}^{1}$-AD.

Main Theorem 2. Conjecture (2) for uniformly degree invariant $\mathbf{\Pi}_{2 n+1}^{1}$ functions is equivalent to $\boldsymbol{\Sigma}_{2 n+2}^{1}-A D$.

We recall some facts and notations (see Sacks [6] which is used as the standard reference in this paper). For each real $x, \omega_{1}^{x}$ denotes the least ordinal $\alpha$ for which $L_{\alpha}[x]$ is admissible. Kleene constructed a $\Pi_{1}^{1}(x)$ complete set $\mathcal{O}^{x}$ with a $\Pi_{1}^{1}(x)$ well founded relation $<_{\mathcal{O}^{x}}$ on $\mathcal{O}^{x}$. The set $\mathcal{O}^{x}$ is the hyperjump of $x$. The height of the ordering $<_{\mathcal{O}^{x}}$ on $\mathcal{O}^{x}$ is exactly $\omega_{1}^{x}$. 
Furthermore, Kleene's construction of $\mathcal{O}^{x}$ is uniform. In other words, the relation $\left\{\left(x, \mathcal{O}^{x}\right) \mid x \in 2^{\omega}\right\}$ is $\Pi_{1}^{1}$. A fact that will be used implicitly is that given reals $x$ and $y, x$ is hyperarithmetic in $y$ (written $x \leq_{h} y$ ) if and only if $x$ is $\Delta_{1}^{1}$ in $y$, and this is in turn equivalent to $x \in L_{\omega_{1}^{y}}[y]$. We work under ZF + DC. As we will only be concerned with Conjecture (2), it will be referred to as the $\leq_{M}$ Conjecture from here on.

\section{The $\leq_{M}$ Conjecture for uniformly order preserving $\Pi_{1}^{1}$ func- tions. Let

$$
\mathcal{F}=\left\{x \mid \forall \alpha<\omega_{1}^{x} \forall a \subseteq \alpha\left(a \in L_{\omega_{1}^{x}} \Rightarrow a \in L_{\alpha+3}[x]\right)\right\} .
$$

$\mathcal{F}$ is a degree invariant $\Sigma_{1}^{1}$ set introduced by H. Friedman [2]. We give a simpler proof of the following result given as Lemma 7.17 in [2].

Lemma 2.1. $\mathcal{F}$ is cofinal in the Turing degrees.

Proof. For any real $z$, let

$$
\mathcal{F}(z)=\left\{x \oplus z \mid \forall \alpha<\omega_{1}^{z} \forall a \subseteq \alpha\left(a \in L_{\omega_{1}^{z}} \Rightarrow a \in L_{\alpha+3}[x \oplus z]\right)\right\}
$$

be a degree invariant $\Sigma_{1}^{1}(z)$ set. Obviously $\mathcal{F}(z)$ is not empty. By the Gandy Basis Theorem relativized to $z$, there is an $x$ such that $\omega_{1}^{x \oplus z}=\omega_{1}^{z}$ and $x \oplus z \in \mathcal{F}(z)$. Then $x \oplus z \in \mathcal{F}$.

The following lemma follows from Lemmas 7.20-7.22 in [2].

Lemma 2.2. If $0^{\sharp}$ does not exist, then $\overline{\mathcal{F}}=2^{\omega}-F$ is cofinal in the Turing degrees.

For $x$ a real and $n \in \omega$, let $x^{[n]}$ be the real such that $x^{[n]}(i)=x(\langle n, i\rangle)$.

THEOREM 2.3. If the $\leq_{M}$ Conjecture holds for uniformly order preserving $\Pi_{1}^{1}$ functions, then $0^{\sharp}$ exists.

Proof. If $0^{\sharp}$ does not exist, then by Lemmas 2.1 and 2.2 , both $\mathcal{F}$ and $\overline{\mathcal{F}}$ are cofinal. For a contradiction, we will define a $\Pi_{1}^{1}$ function $G$ that is uniformly order preserving such that $\left\{x \mid G(x)=\mathcal{O}^{\mathcal{O}^{x}}\right\}$ and $\{x \mid G(x)=$ $\left.\mathcal{O}^{\mathcal{O}^{x}}\right\}$ are both cofinal in the Turing degrees.

Let $P(x, y)$ be an arithmetic predicate such that

$$
x \in \overline{\mathcal{F}} \Leftrightarrow \forall y P(x, y) .
$$

Claim 2.4. If $x \leq_{T} y$ are such that $x \in \mathcal{F}$ and $y \in \overline{\mathcal{F}}$, then $\mathcal{O}^{x} \leq_{h} y$.

As $y \notin \mathcal{F}$, there are $\alpha<\omega_{1}^{y}$ and $a \subseteq \alpha$ with $a \in L_{\omega_{1}^{y}} \backslash L_{\alpha+3}[y]$. Clearly, $\alpha \geq \omega$. As $x \leq_{T} y, L_{\alpha+3}[x] \subseteq L_{\alpha+3}[y]$ and thus $a \notin L_{\alpha+3}[x]$. As $x \in \mathcal{F}$, $\omega_{1}^{x} \leq \alpha<\omega_{1}^{y}$. By $x \leq_{T} y$ again, $\mathcal{O}^{x} \leq_{h} y$.

By Claim 2.4 if $x \leq_{T} y$ are such that $x \in \mathcal{F}$ and $y \in \overline{\mathcal{F}}$, then $\mathcal{O}^{\mathcal{O}^{x}} \leq_{T} \mathcal{O}^{y}$.

Now define $G(x)=y$ as follows: 
(1) $y^{[0]}=\langle 0\rangle^{\wedge} \mathcal{O}^{\mathcal{O}^{x}} \wedge x \in \overline{\mathcal{F}}$ or $y^{[0]}=\langle 1\rangle^{\wedge} \mathcal{O}^{\mathcal{O}^{\mathcal{O}^{x}}} \wedge \exists v \leq_{T} y^{[0]} \neg P(x, v)$. Thus $y^{[0]}$ gives a $\Pi_{1}^{1}$ differentiation between $x \in \overline{\mathcal{F}}$ and $x \in \mathcal{F}$.

(2) $y^{[1]}=\mathcal{O}^{x}$.

(3) If $\Phi_{e}^{x}$ is partial then let $y^{[e+2]}=\emptyset$.

(4) If $\Phi_{e}^{x}$ is total and equal to $u$, the following three cases differentiate in a $\Pi_{1}^{1}$ way between $u \in \mathcal{F}$ and $x \in \overline{\mathcal{F}}, u, x \in \mathcal{F}$, and $u \in \overline{\mathcal{F}}$ for all $u \leq_{T} x$ :

(a) $y^{[0]}(0)=0 \wedge \exists v \leq_{T} y^{[1]} \neg P(u, v) \wedge y^{[e+2]}=\langle 1\rangle^{\wedge} \mathcal{O}^{\Phi_{i}^{y^{[1]}}}$ where $i$ is the least index so that $\mathcal{O}^{\mathcal{O}^{u}}=\mathcal{O}^{\Phi_{i}^{y^{[1]}}}$, or

(b) $y^{[0]}(0)=1 \wedge \exists v \leq_{T} y^{[1]} \neg P(u, v) \wedge y^{[e+2]}=\langle 1\rangle^{\wedge} \mathcal{O}^{\mathcal{O}^{u}}$, or

(c) $\forall v \leq_{T} y^{[1]} P(u, v) \wedge y^{[e+2]}=\langle 0\rangle^{\wedge} \mathcal{O}^{\mathcal{O}^{u}}$.

$G(x)$ is obviously $\Pi_{1}^{1}$.

Claim 2.5. If $x \in \mathcal{F}$ then $G(x) \equiv_{T} \mathcal{O}^{\mathcal{O}^{\mathcal{O}^{x}}}$.

Clearly $x \in \mathcal{F}$ implies $\mathcal{O}^{\mathcal{O}^{x}} \leq_{T} G(x)$ and $G(x)^{[0]} \oplus G(x)^{[1]} \leq_{T} \mathcal{O}^{\mathcal{O} x}$.

Given $e<\omega, \mathcal{O}^{\mathcal{O}^{x}}$ can uniformly decide whether $\Phi_{e}^{x}$ is total. Suppose that $\Phi_{e}^{x}$ is total. To calculate $G(x)^{[e+2]}(n)$, one verifies clauses (4b, 4c) above. But the predicate $\forall v \leq_{T} y^{[1]} P(u, v)$ is $\Delta_{1}^{1}\left(\mathcal{O}^{x}\right)$, hence recursive in $\mathcal{O}^{\mathcal{O}^{x}}$. Once this predicate is decided, $\mathcal{O}^{\mathcal{O}^{x}}$ may use recursive functions $f$ and $g$, where $u=\Phi_{e}^{w} \rightarrow \mathcal{O}^{\mathcal{O}^{u}}=\Phi_{f(e)}^{\mathcal{O}^{\mathcal{O}^{w}}}$ and $u=\Phi_{e}^{w} \rightarrow \mathcal{O}^{\mathcal{O}^{\mathcal{O}^{u}}}=\Phi_{g(e)}^{\mathcal{O}^{\mathcal{O}^{w}}}$, to finish the calculation.

Claim 2.6. If $x \in \overline{\mathcal{F}}$ then $G(x) \equiv_{T} \mathcal{O}^{\mathcal{O}^{x}}$.

This is similar to the above claim, except for the final step calculating $G(x)^{[e+2]}(n)$.

Now $\mathcal{O}^{\mathcal{O}^{x}}$ is able to decide whether (4a) or (4c) holds, as in the above claim. If (4c) holds, the calculation is the same. If (4a) holds, then $u \in \mathcal{F}$. By Claim 2.4. $\mathcal{O}^{u} \leq_{h} x$ and thus $\mathcal{O}^{\mathcal{O}^{u}} \leq_{T} \mathcal{O}^{x}=G(x)^{[1]}$. So $i$ exists. Moreover, the search for $i$ is a procedure uniformly $\Pi_{1}^{1}\left(\mathcal{O}^{x}\right)$. Hence $\mathcal{O}^{\mathcal{O}^{x}}$ uniformly computes $G(x)^{[e+2]}(n)$.

It follows from the above two claims that $G$ is degree invariant. Moreover, $G$ preserves $\leq_{T}$ by Claim 2.4 .

To show that $G$ is uniformly order preserving, let $h$ be a recursive function such that $\forall x, y, e\left(x=\Phi_{e}^{y} \rightarrow \mathcal{O}^{x}=\Phi_{h(e)}^{\mathcal{O}^{y}}\right)$. In addition, let $s$ be recursive with

$$
\forall x, y, e, i\left(x=\Phi_{e}^{y} \rightarrow \Phi_{i}^{x}=\Phi_{s(e, i)}^{y}\right) .
$$

Suppose that $x=\Phi_{e}^{y}$. Then

(1) $G(x)^{[0]}=G(y)^{[e+2]}$, 
(2) $G(x)^{[1]}=\Phi_{h(e)}^{G(y)^{[1]}}$,

(3) $G(x)^{[i+2]}=G(y)^{[s(e, i)+2]}$.

Hence $G$ is as desired.

The above proof easily relativizes to any real $x$ to guarantee the existence of $x^{\#}$. Since Harrington [1] has shown that the existence of sharps implies $\Pi_{1}^{1}$-TD, we have

Main Theorem 1. If the $\leq_{M}$ Conjecture holds for $\Pi_{1}^{1}$ functions which are uniformly order preserving, then $\Pi_{1}^{1}-T D$ is true.

\section{The $\leq_{M}$ Conjecture for $\Pi_{2 n+1}^{1}$ functions and $\Sigma_{2 n+2}^{1}$-TD}

LEMMA 3.1. $\Pi_{2 n+1}^{1}$-uniformization and $\Delta_{2 n+2}^{1}-T D$ imply $\Sigma_{2 n+2}^{1}$-TD for $n \in \omega$.

Proof. Let $A \in \Sigma_{2 n+2}^{1}$ be degree invariant and $\leq_{T}$-cofinal. Define

$$
R(x, y) \Leftrightarrow x \leq_{T} y \wedge y \in A .
$$

So $R(x, y) \in \Sigma_{2 n+2}^{1}$. Note that $\Pi_{2 n+1}^{1}$-uniformization implies $\Sigma_{2 n+2}^{1}$-uniformization. Let $F \in \Sigma_{2 n+2}^{1}$ uniformize $R$. Then $F$ is actually a $\Delta_{2 n+2}^{1}$ function.

Define

$$
B=\left\{u \mid \exists x \leq_{T} u, y \equiv_{T} u(F(x)=y)\right\} .
$$

Then $B$ is $\Delta_{2 n+2}^{1}$, degree invariant and $\leq_{T}$-cofinal. Moreover, $B \subseteq A$. By $\Delta_{2 n+2}^{1}$ TD, $B$ contains a cone of Turing degrees. Hence so does $A$.

Corollary 3.2. $\Delta_{2}^{1}$-TD implies $\Sigma_{2}^{1}-T D$.

Proof. As $\Pi_{1}^{1}$-uniformization is a theorem of ZFC, the corollary follows immediately from Lemma 3.1 .

We prove the next result for the lightface version. The proof for the boldface version follows with obvious changes.

Theorem 3.3. Assume $\Pi_{2 n+1}^{1}$-uniformization. If the $\leq_{M}$ Conjecture holds for uniformly degree invariant $\Pi_{2 n+1}^{1}$ functions, then $\Sigma_{2 n+2}^{1}$-TD holds.

Proof. Let $A \in \Delta_{2 n+2}^{1}$, and suppose $P, Q \in \Pi_{2 n+1}^{1}$ are such that

$$
x \in A \Leftrightarrow \exists y P(x, y) \Leftrightarrow \forall y \neg Q(x, y) .
$$

Let $R(x, y) \Leftrightarrow P(x, y) \vee Q(x, y)$. By $\Pi_{2 n+1}^{1}$-uniformization, let $F \in \Pi_{2 n+1}^{1}$ uniformize $R$. Define $J_{0}(x)=z$ if and only if $z^{[0]}=F(x)$ and

$$
\forall e\left(\left(\Phi_{e}^{x} \text { is total } \rightarrow z^{[e+1]}=F\left(\Phi_{e}^{x}\right)\right) \wedge\left(\Phi_{e}^{x} \text { is partial } \rightarrow z^{[e+1]}=\emptyset\right)\right) .
$$

Obviously $J_{0} \in \Pi_{2 n+1}^{1}$ is total. Moreover, $J_{0}$ is uniformly order preserving. To see this, let $f$ be a recursive function such that

$$
\forall e, x_{0}, x_{1}\left(x_{0}=\Phi_{e}^{x_{1}} \rightarrow \forall i\left(\Phi_{f(e, i)}^{x_{1}} \simeq \Phi_{i}^{x_{0}}\right)\right) .
$$


Suppose $x_{0}=\Phi_{e}^{x_{1}}$. Then $\left(J_{0}\left(x_{0}\right)\right)^{[0]}=\left(J_{0}\left(x_{1}\right)\right)^{[e]}$ and $\left(J_{0}\left(x_{0}\right)\right)^{[i+1]}=$ $\left(J_{0}\left(x_{1}\right)\right)^{[f(e, i)+1]}$. Thus $J_{0}\left(x_{0}\right)$ may be effectively computed from $J_{0}\left(x_{1}\right)$.

Let $g$ be a recursive function such that $x_{0}=\Phi_{e}^{x_{1}} \rightarrow J_{0}\left(x_{0}\right)=\Phi_{g(e)}^{J_{0}\left(x_{1}\right)}$.

Define $J(x)=x \oplus z_{0} \oplus z_{1}$ if and only if $z_{0}=J_{0}(x)$ and

$$
\left(P\left(x, z_{0}^{[0]}\right) \wedge z_{1}=\emptyset\right) \vee\left(Q\left(x, z_{0}^{[0]}\right) \wedge z_{1}=\langle 1\rangle^{\wedge}\left(x \oplus z_{0}\right)^{\prime}\right) .
$$

Note that $J \in \Pi_{2 n+1}^{1}$. We claim that $J$ is uniformly degree invariant. To see this, let $h$ be a recursive function such that $x_{0}=\Phi_{e}\left(x_{1}\right) \rightarrow\left(x_{0} \oplus J_{0}\left(x_{0}\right)\right)^{\prime}=$ $\Phi_{h(e)}^{\left(x_{1} \oplus J_{0}\left(x_{1}\right)^{\prime}\right.}$. For each $e$, let $t(e)$ be the index of the procedure $\Psi$ defined by:

1. $\left(\Psi^{z}\right)^{[0]}=\Phi_{e}^{z^{[0]}}$ and $\left(\Psi^{z}\right)^{[1]}=\Phi_{g(e)}^{z^{[1]}}$.

2. If $z^{[2]}(0)=0$ then $\left(\Psi^{z}\right)^{[2]}=\emptyset$. Otherwise $\left(\Psi^{z}\right)^{[2]}=\langle 1\rangle^{\wedge} \Phi_{h(e)}^{w}$, where $w$ is such that $\langle 1\rangle^{\wedge} w=z^{[2]}$.

If the $\leq_{M}$ Conjecture holds for uniformly degree invariant $\Pi_{2 n+1}^{1}$ functions, then eventually $J$ is either $x \mapsto x \oplus J_{0}(x)$ or $x \mapsto x \oplus J_{0}(x) \oplus\left(x \oplus J_{0}(x)\right)^{\prime}$. Hence $A$ either contains or avoids a cone of Turing degrees.

Thus we have $\Delta_{2 n+2}^{1}$-TD. Now $\Sigma_{2 n+2}^{1}$-TD follows from Lemma 3.1 .

Main Theorem 2. Let $n \geq 0$. The $\leq_{M}$ Conjecture for uniformly degree invariant $\boldsymbol{\Pi}_{2 n+1}^{1}$ functions is equivalent to $\boldsymbol{\Sigma}_{2 n+2}^{1}-A D$.

Proof. An analysis of Theorem 1 in Steel [8] shows that $\boldsymbol{\Sigma}_{2 n+2}^{1}$ AD (in fact $\boldsymbol{\Delta}_{2 n+2}^{1}$-AD) implies the $\leq_{M}$ Conjecture for uniformly degree invariant $\Pi_{2 n+1}^{1}$ functions. We show the converse by induction on $n$ : First note that if $n=0$, then $\boldsymbol{\Pi}_{1}^{1}$-uniformization is the Kondo-Addison Theorem, so that by Theorem 3.3, $\boldsymbol{\Sigma}_{2}^{1}$-TD holds. Now assume by induction that $\boldsymbol{\Sigma}_{2 n}^{1}$-TD is true. Woodin (unpublished) has shown that over ZFC, for $k \geq 1, \boldsymbol{\Sigma}_{2 k}^{1}$-TD is equivalent to $\boldsymbol{\Sigma}_{2 k}^{1}$-AD, and Moschovakis [5, Chapter 6] has shown that $\Pi_{2 k+1^{-}}^{1}$ uniformization is a consequence of $\boldsymbol{\Sigma}_{2 k}^{1}$-AD. Thus $\boldsymbol{\Pi}_{2 n+1}^{1}$-uniformization holds and so Theorem 3.3 yields $\boldsymbol{\Sigma}_{2 n+2}^{1}$-TD, hence $\boldsymbol{\Sigma}_{2 n+2}^{1}$-AD.

The following corollary gives the consistency strength of the $\leq_{M}$ Conjecture.

COROLlary 3.4. If it is consistent that the $\leq_{M}$ Conjecture holds for uniformly degree invariant $\Pi_{1}^{1}$ functions, then it is consistent that there is a Woodin cardinal.

Proof. The hypothesis and Theorem 3.3 imply that $\Pi_{2}^{1}$-TD is consistent. Woodin has shown that $\Pi_{2}^{1}$-TD is equiconsistent with the existence of a Woodin cardinal.

REMARK. We do not know if Main Theorem 1 may be strengthened to $\boldsymbol{\Delta}_{2}^{1}$-TD (hence $\boldsymbol{\Delta}_{2}^{1}$-AD). If this is true, then by Steel [8] it will give a characterization of the $\leq_{M}$ Conjecture for uniformly order preserving $\boldsymbol{\Pi}_{1}^{1}$ 
functions. In general, one would like to understand better the role of order preserving functions in the study of the $\leq_{M}$ Conjecture. For example, it is not clear if Corollary 3.4 applies to functions which are order preserving.

Acknowledgments. The research of Chong and Wang was supported in part by NUS grant WBS 146-000-054-123, and Yu was supported by NSF of China No. 10701041 as well as Research Fund for Doctoral Program of Higher Education No. 0070284043.

\section{References}

[1] L. Harrington, Analytic determinacy and $0^{\sharp}$, J. Symbolic Logic 43 (1978), 685-693.

[2] R. Mansfield and G. Weitkamp, Recursive Aspects of Descriptive Set Theory, Oxford Logic Guides 11, Oxford Univ. Press, New York, 1985.

[3] D. A. Martin, The axiom of determinateness and reduction principles in the analytical hierarchy, Bull. Amer. Math. Soc. 74 (1968), 687-689.

[4] —, Borel determinacy, Ann. of Math. (2) 102 (1975), 363-371.

[5] Y. N. Moschovakis, Descriptive Set Theory, Stud. Logic Found. Math. 100, NorthHolland, Amsterdam, 1980.

[6] G. E. Sacks, Higher Recursion Theory, Perspectives in Math. Logic, Springer, Berlin, 1990.

[7] T. A. Slaman and J. R. Steel, Definable functions on degrees, in: Cabal Seminar 81-85, Lecture Notes in Math. 1333, Springer, Berlin, 1988, 37-55.

[8] J. R. Steel, A classification of jump operators, J. Symbolic Logic 47 (1982), 347-358.

C. T. Chong

Department of Mathematics

Faculty of Science

National University of Singapore

Lower Kent Ridge Road

Singapore 117543

E-mail: chongct@math.nus.eud.sg

Liang $\mathrm{Yu}$

Institute of Mathematical Sciences

Nanjing University

Nanjing, Jiangsu Province 210093, P.R. China

E-mail: yuliang.nju@gmail.com
Wei Wang

Department of Philosophy Sun Yat-sen University 135 Xingang Xi Road Guangzhou 510275, P.R. China E-mail: wwang.cn@gmail.com

Received 20 December 2008; in revised form 30 October 2009 